\title{
Comparison of ovarian crescent sign and risk of malignancy index for prediction of ovarian malignancy in adnexal masses
}

\author{
Abha Sharma, Richa Sharma*, Ashita Gulati \\ Department of Obstetrics and Gynaecology, UCMS and GTB Hospital, Delhi, India \\ Received: 28 August 2020 \\ Accepted: 18 December 2020 \\ *Correspondence: \\ Dr. Richa Sharma, \\ E-mail: gautamdrricha1@gmail.com \\ Copyright: (c) the author(s), publisher and licensee Medip Academy. This is an open-access article distributed under \\ the terms of the Creative Commons Attribution Non-Commercial License, which permits unrestricted non-commercial \\ use, distribution, and reproduction in any medium, provided the original work is properly cited.
}

\begin{abstract}
Background: Objective of the study was to evaluate ovarian crescent sign (OCS) as a sonographic parameter for prediction of ovarian cancer in adnexal masses suspicious of ovarian malignancy and to compare it with risk of malignancy index (RMI).

Methods: Presence of OCS and calculation of RMI was done for 50 cases of adnexal masses scheduled to undergo surgery taking histopathology as gold standard.

Results: $18 \%$ (9/50) of adnexal masses were malignant. OCS was absent in all malignant lesions, giving a sensitivity and negative predictive value of $100 \%$. OCS was present in 33/41 of benign masses (specificity $80.4 \%$ ). Relation of OCS to mass size $<10 \mathrm{~cm}$ and menopausal status was significant $(\mathrm{p}<0.001)$. RMI $\geq 200$ could not diagnose malignancy in $4 / 9$ cases (sensitivity $55.5 \%$ ). RMI had specificity and negative predictive value of $95.1 \%$ and $90.7 \%$ respectively. Combining OCS and RMI had a lower specificity. Sequential application using OCS as first node and RMI as second node failed to diagnose $44.4 \%$ (4/9) cases as malignant.

Conclusions: OCS is cheaper, easy to perform and appears to be a better test than RMI to differentiate between benign and early-stage malignant ovarian tumors. It can be used for triaging patient for referral.
\end{abstract}

Keywords: Ovarian crescent sign, Risk of malignancy index, Ovarian malignancy, Adnexal mass

\section{INTRODUCTION}

Ovarian neoplasms presenting as adnexal masses is not an uncommon finding in gynecological practice with rate of malignancy being 4-24\% for premenopausal and 39$63 \%$ for postmenopausal patients. ${ }^{1}$ However, ovarian malignancy is mostly diagnosed at late stages (epithelial ovarian cancer stage $3-4=70 \%$ with low 5 year survival rate of $20-25 \%$ as compared to stage 1 with $90 \% 5$ year survival rate), thus jeopardizing the patient's survival. ${ }^{2}$ The need of the hour is to suspect and diagnose ovarian malignancy with confidence at an early stage as diagnosis is vital for triaging the patients. Studies have shown that the patients who are treated by gynaecological oncologists at the dedicated centers are more likely to undergo a complete surgical staging and have decreased morbidity and mortality and improved 5-year survival rates. ${ }^{3}$ Also the patients with definite diagnosis of benign tumors can safely undergo conservative and less radical surgery at peripheral centers, thus decreasing the load on tertiary centers. ${ }^{4}$ Several diagnostic methods for pelvic masses have been reported such as serum tumors markers, transabdominal and transvaginal sonography, color doppler ultrasound and other imaging modalities like CT scan, MRI and PET scan. The value of serum CA-125 level as a screening method is limited by its inability to detect ovarian cancer in early stages (only 25$50 \%$ of early ovarian cancers show raised serum CA-125 levels) and increase in non-gynecologic cancers and benign conditions. The specificity of ultrasonography is $73-95 \% .{ }^{1,2}$ Various scoring systems are described in the literature, which involves the ultrasonic morphological parameters. Color doppler RI and PI values are helpful but the disadvantage is that, many times it may overlap in 
benign and malignant ovarian tumors and also it requires trained specialists and specialized machines. ${ }^{2,5}$ The RMI is a scoring system based on menopausal status, ultrasound and serum concentrations of CA-125. Using a cut off of 200 to indicate malignancy, sensitivity was $85 \%$ and specificity was $97 \% .^{6}$

A new parameter, the OCS has been introduced by Hillaby in 2004, which refers to the presence of normal ovarian tissue adjacent to adnexal mass. ${ }^{7}$ This is a simplified approach to the morphological analysis of ovarian tumors for preoperative diagnosis of ovarian malignancy. OCS sensitivity is $91 \%$, specificity $84 \%$, positive predictive value $73 \%$ and negative predictive value of $95 \%$. There have been conflicting reports in the literature regarding its efficacy.

So, this study was undertaken to establish its efficacy in the diagnosis of ovarian tumors.

\section{METHODS}

This was a cross-sectional study done at Guru Teg Bahadur hospital, Delhi from July 2016 to June 2018. Total 50 women with adnexal masses, who underwent surgical interventions at our institution were enrolled for the study. The exclusion criteria were-patients of obvious ovarian malignancy with secondaries, extrauterine pregnancy, gestational trophoblastic neoplasia and obvious tubal masses with clearly defined separate ovaries. Ethical clearance was obtained from the institutional ethical committee All the patients were managed as per protocol Table 1 .

RMI was calculated for each patient as per criteria by Tingulstad et al $(1996)^{8}$ : RMI=U x M x serum CA125 level where $\mathrm{U}=$ ultrasound score-1 or 4 and $\mathrm{M}=$ Menopausal status-1 or 4 . The RMI value $\geq 200$ was considered significant for malignancy.

Menopausal status was defined as more than one year of amenorrhea or age $>50$ years in women who had hysterectomy. Premenopausal status was given a score of 1 whereas postmenopausal status was given a score of 4 . The absolute value of serum CA-125 (U/ml) was noted. Ultrasound features noted for RMI were: multilocular cystic lesion, solid areas, bilateral lesions, ascites and intra-abdominal metastasis. The presence of each was given a score of 1. A total ultrasonic score (U) was calculated for each patient. A score of $0-1$ was given $U=1$ and a score of $\geq 2$ was given $U=4$.

Ovarian crescent sign was defined as the presence of normal ovarian tissue adjacent to the tumor, which was identified as hypoechogenic tissue with or without follicles adjacent to the cyst wall enclosed within the ovarian capsule, which could not be separated from the cyst wall by applying moderate amount of pressure Table 2,3. OCS was mentioned as being present or absent.
Absence of ovarian crescent was indicative of malignancy.

Histopathologic diagnosis was considered as gold standard. On the basis of histopathology of the masses, the subjects were divided into two groups: benign and malignant. All the statistical analyses were carried out using SPSS version 13.0. Comparison of the data was done by unpaired student t-test for those who follow normal distribution. Log based 10 transformation was used for RMI and serum CA-125 levels to make them normal and then apply student t-test. For qualitative dataPearson's chi-square test was used if the asymptotic was valid; else exact significance was calculated. $\mathrm{p}<0.05$ was taken as significant in all the tests.

\section{RESULTS}

Eighteen percent (9/50) of patients had malignant lesions and $82 \%(41 / 50)$ had benign lesions. No borderline tumors were reported. Amongst malignant lesions, incidence of epithelial ovarian tumors was $55.5 \%$, serous cystadenocarcinoma being the most common primary malignant tumor $(44.4 \%, 4 / 9)$. Most of the patients were early stage one ovarian cancers $(66.6 \%, 6 / 9)$. Only $2 / 9$ $(22.2 \%)$ were stage III ovarian carcinomas. Amongst benign tumors, serous and mucinous cystadenomas account for $68.3 \%(28 / 41)$ of cases with a significant number of endometriomas $(19.5 \%, 8 / 41)$ (Table 1$)$.

Table 1: Histopathology of tumors.

\begin{tabular}{|lll|}
\hline Histopathology of tumors & No. & $\%$ \\
\hline Benign tumor & & \\
\hline Serous cystadenoma & 21 & 51.2 \\
\hline Endometrioma & 8 & 19.5 \\
\hline Mucinous cystadenoma & 7 & 17.1 \\
\hline Dermoid & 3 & 7.3 \\
\hline Brenner's & 1 & 2.4 \\
\hline Fibrothecoma & 1 & 2.4 \\
\hline Total & 41 & 100.0 \\
\hline Malignant tumor & & \\
\hline $\begin{array}{l}\text { Papillary serous } \\
\text { cystadenocarcinoma }\end{array}$ & 4 & 44.4 \\
\hline Mucinous adenocarcinoma & 1 & 11.11 \\
\hline $\begin{array}{l}\text { Granulosa cell tumor } \\
\text { Dysgerminoma }\end{array}$ & 1 & 11.11 \\
\hline $\begin{array}{l}\text { Sex cord stromal tumor of } \\
\text { unknown histogenesis }\end{array}$ & 1 & 11.11 \\
\hline Krukenberg's tumor & 1 & 11.11 \\
\hline Total & 1 & 11.11 \\
\hline
\end{tabular}

Both the groups were matching with respect to parity, socioeconomic status and religion. Mean age was 6 years more in malignant group as compared to benign ( $42 \pm 14$ years vs $36 \pm 15$ years), p-value however being not significant. Postmenopausal status was twice as frequent in malignant group as compared to benign group (44.4\% 
vs $22 \%$ ), though it was statistically not significant $(\mathrm{p}>0.05)$.

OCS was absent in all the malignant cases (100\%), whereas it was present in $80.5 \%$ of benign cases. None of the patients with visualization of OCS were found to be malignant (Table 2). Histopathology of the 8 benign masses with absent OCS was as follows-3 mucinous cystadenomas, 2 serous cystadenomas, and one case each of Brenner's tumor, teratoma and fibro thecoma ovary.

Table 2: OCS and the nature of adnexal mass.

\begin{tabular}{|lll|}
\hline OCS & Benign $n=41(\%)$ & Malignant $n=9(\%)$ \\
\hline Present & $33(80.4)$ & 0 \\
\hline Absent & $8(19.5)$ & $9(100)$ \\
\hline
\end{tabular}

Evaluation of all the benign tumors with absent OCS, a significant relationship was seen between detection of OCS with size of the tumor and postmenopausal status (Table 3). In benign group, ovarian crescent sign was present in $93.9 \%$ of patients with size $<20 \mathrm{~cm}$. On further looking deeper into the group, OCS was present in all the patients with size $<10 \mathrm{~cm}$ ( 23 out of 23 ), but was absent in 6 out of $8(75 \%)$ ovarian masses with size $\geq 20 \mathrm{~cm}$ (Table 3). OCS was absent in significant number of postmenopausal women ( 5 out of $9,55.6 \%$ ) with benign tumors as compared to premenopausal women (3 out of $32,9.4 \%) \quad(\mathrm{p}<0.001)$, but all these postmenopausal women had large ovarian tumors also $(>15 \mathrm{~cm}$ size) (Table 3).

RMI $\geq 200$ missed $44.44 \%$ (4/9) of malignant patients and falsely diagnosed $4.9 \% \quad(2 / 41)$ benign masses as malignant (Table 4).
Table 3: Distribution of ovarian crescent sign in benign tumors.

\begin{tabular}{|c|c|c|c|c|}
\hline $\begin{array}{l}\text { Ovarian } \\
\text { crescent sign }\end{array}$ & $\mathbf{N}$ & $\begin{array}{l}\text { OCS+ } \\
\text { n }(\%)\end{array}$ & $\begin{array}{l}\text { OCS- } \\
\text { n }(\%)\end{array}$ & $\begin{array}{l}\mathbf{P} \\
\text { value }\end{array}$ \\
\hline \multicolumn{5}{|l|}{ Tumor size (cm) } \\
\hline$<10$ & 23 & $23(100)$ & 0 & \multirow{3}{*}{$<0.001$} \\
\hline $10-19$ & 10 & $8(80)$ & $2(20)$ & \\
\hline$\geq 20$ & 8 & $2(25)$ & $6(75)$ & \\
\hline \multicolumn{5}{|c|}{ Menopausal status } \\
\hline Premenopausal & 32 & $29(90.6)$ & $3(9.4)$ & \multirow{2}{*}{$<0.001$} \\
\hline Postmenopausal & 9 & $4(44.4)$ & $5(55.6)$ & \\
\hline
\end{tabular}

Table 4: RMI in both the groups.

\begin{tabular}{|lll|}
\hline RMI & Benign $\mathbf{n = 4 1}(\%)$ & Malignant $\mathbf{n = 9}(\%)$ \\
\hline $\mathbf{2 0 0}$ & $39(95.1)$ & $4(44.4)$ \\
$\mathbf{2 2 0 0}$ & $2(4.9)$ & $5(55.5)$ \\
\hline
\end{tabular}

Absence of ovarian crescent sign had a very high sensitivity (100\%) and negative predictability (100\%) with an acceptable specificity of $80.4 \%$ for diagnosing ovarian malignancy as compared to $\mathrm{RMI} \geq 200$ which had a low sensitivity of $55.5 \%$, negative predictive value of $90.7 \%$ but good specificity of $95.1 \%$ (Table 5).

An attempt was made to see whether both tests taken together or sequentially improved the probability of diagnosis of ovarian malignancy.

Applying both the tests simultaneously for detection of ovarian malignancy increased the sensitivity of RMI to $100 \%$ (i.e., equal to sensitivity of absence of OCS) but had a specificity of $76.5 \%$ which was lower than both RMI and absent OCS (Table 5).

Table 5: Comparison of the predictive accuracy of RMI and OCS and combination of both for diagnosing ovarian malignancy.

\begin{tabular}{|lllll|}
\hline Variables & $\begin{array}{l}\text { Sensitivity } \\
(\%)\end{array}$ & $\begin{array}{l}\text { Specificity } \\
(\%)\end{array}$ & $\begin{array}{l}\text { Positive predictive } \\
\text { value (\%) }\end{array}$ & $\begin{array}{l}\text { Negative predictive } \\
\text { value }(\%)\end{array}$ \\
\hline Risk of malignancy index & 55.6 & 95.1 & 71.4 & 90.7 \\
\hline $\begin{array}{l}\text { Absence of ovarian } \\
\text { crescent sign }\end{array}$ & 100 & 80.4 & 52.9 & 100 \\
\hline Combined & 100 & 76.5 & & \\
\hline
\end{tabular}

On sequential use of these tests, OCS was taken as first node because $\mathrm{RMI} \geq 200$ missed $44.4 \%$ (4/9) of malignant cases. Absence of OCS was $100 \%$ sensitive. So RMI $\geq$ 200 was applied to all the patients with absence of OCS. This model too missed $44.4 \%$ (4/9) of malignant cases. So, the sequential use was not found to be beneficial Table 4.

\section{DISCUSSION}

This study was undertaken to evaluate the role of OCS in differentiating between malignant and benign ovarian tumors, inability to visualize normal ovarian tissue adjacent to ovary (absent OCS), being indicative of malignancy.

\section{Ovarian crescent sign}

In the present study, OCS was absent in all the patients with malignant lesions (9/9) giving it a sensitivity and negative predictive value of $100 \%$. This was similar to other studies by Hillaby, Yazbek and Kushtagi et al (sensitivity: 96\%, 100\%, 90.9\%; NPV: 95\%, 100\%, 97.4\%). ${ }^{7,9,10}$ High sensitivity and NPV of absent OCS in 
identifying malignancy has also been confirmed in the multicentric study done in a subgroup of patients of international ovarian tumor analysis (IOTA) phase 2 study by Van Holsbeke et al (sensitivity 92\%, NPV $92 \%) .{ }^{11}$ There was no false positive in our study but most of false positives in other studies have been reported in borderline tumors. Hillaby et al reported one false positive OCS in a case of benign endometrioma with a small focus of clear cell carcinoma. ${ }^{7}$ False positive OCS could be seen in 18/305 (6\%) of the invasive and in 14/86 $(16 \%)$ of the borderline tumors in IOTA subgroup study. ${ }^{11}$ Also, in the study by Yazbek et al, OCS was present in $18 / 35(51.4 \%)$ of borderline tumors. There was no borderline tumor found in our study. ${ }^{9}$

The specificity of absent OCS was $80.4 \%$ in the study with $8 / 41(19.5 \%)$ benign tumors showing absence of healthy ovarian tissue. This is almost similar to the study by Hillaby, Yazbek and Kushtagi et al, which showed specificity of 76,93 and $77.6 \%$ respectively. ${ }^{7,9,10}$ The IOTA phase 2 subgroup study on OCS, however, had a low specificity of $42 \%$ for absent OCS. ${ }^{11}$ The authors argued that the poor performance of OCS in their study might be due to the lack of specific training in assessing it or due to the difference in the study population.

Absent OCS for a malignant tumor had a low positive predictive value of $52.9 \%$ similar to Hillaby $(56 \%)$, Yazbek et al $(56 \%)$ and IOTA phase 2 subgroup study $(43 \%)^{7,9,11}$ This is due to high number of benign tumors with absent OCS (8/41).

Amongst the 8 benign masses with absence of OCS, six masses had a size $\geq 20 \mathrm{~cm}$ and two had sizes between 10 $19 \mathrm{~cm}$. Moreover, 5 of these patients were postmenopausal, making investigation more difficult. Such a large size would cause inability to see a small area of normal ovarian tissue or it could cause excessive stretching of ovarian tissue over the tumor marking the crescent difficult to detect. In the multicentric IOTA subgroup study too, the OCS was absent more in larger size tumors $(\mathrm{p}<0.001)$ in both benign and malignant group. ${ }^{11}$

The IOTA subgroup study and Kushtagi et al found a significant relationship between menopausal status and OCS, with lower rate of visualization of ovarian crescent in postmenopausal as compared to premenopausal women in benign tumors ( $p$ value<0.05). ${ }^{10,11}$ In the present study too, amongst the benign group, OCS was absent in 55.6\% (5/9) of postmenopausal patients as compared from only $9.4 \%$ (3/32) of premenopausal patients but all these patients had big size $(>15 \mathrm{~cm})$ tumors also. However, in all 3 postmenopausal patients in whom tumor size was $\leq 10 \mathrm{~cm}$, OCS could be seen. Therefore, probably tumor size is more important determinant for detection of OCS than the lesser ovarian volume in postmenopausal females.
On the other hand, all the masses with presence of OCS were benign. This significant finding has been confirmed by the multicentric IOTA phase 2 subgroup study too. ${ }^{11} \mathrm{It}$ has clinical implication in the triaging of patients by offering the management to the patients with presence of OCS at the peripheral centers and referring the patients with absent OCS to higher centers for further investigations and appropriate management.

\section{Risk of malignancy index}

Morgante et al found that RMI 2 performed better than RMI1. ${ }^{1}$ So RMI 2 (Tingulstad et al) was used for this study. $^{2}$ The sensitivity of RMI for detection of malignancy was variously reported from $71-85 \%$ by Jacobs and Tingulstad et al, but these results were obtained with $64 \%$ and $57 \%$ cases respectively being in late stage of ovarian carcinoma (>stage II). ${ }^{6,8}$ For detection of early carcinoma ovary (stage I and II), it was reported to be only $41 \%$. In this study too, the sensitivity of RMI $\geq 200$ to predict malignancy was found to be only $55.6 \%$ due to high number of patients $(66.6 \%)$ in stage I disease. Here, RMI<200 was found in 4/9 (44.4\%) of malignant masses-all stage I tumors (two sex cord stromal tumors, one mucinous cystadenocarcinoma, one papillary cystadenocarcinoma) and serum levels serum CA-125 levels are not high in non-epithelial malignant tumors and stage I tumors and RMI is highly dependent on the absolute serum CA-125 levels in the study by Tingulstad et al, among the 22 patients with stage I and II ovarian cancer, $13(59 \%)$ had an RMI score of $<200{ }^{8}$

The specificity of RMI for detection of malignancy in this study was $95.1 \%$, which was well comparable with other studies (Jacobs 97\%, Tingulstad 71-80\%, Yazbek $92 \%$, and Kushtagi et al-85.8-89.8\%). ${ }^{6,8-10}$ RMI $\geq 200$ was found in $2 / 41(4.9 \%)$ cases in benign group; both of which were endometriomas with high level of serum CA125 levels. In study by Yazbek et al, the falsest positive results with RMI were also found in women with endometriotic cysts, which contributed to $87.5 \%$ of high readings in benign pathology. ${ }^{9}$

The positive predictive value of $71.4 \%$ in this study was slightly lower than other studies by Tingulstad et al (89\%), but higher than study by Yazbek $(50 \%)$ and Kushtagi et al 53.3-61.5\%.$^{8-10}$ The negative predictive value of $91 \%$ in this study was well comparable to Tingulsted (88-91\%), Yazbek (99\%) and Kushtagi et al $(93.3-95.3 \%) .^{8-10}$

\section{Comparison of OCS and RMI}

Results of our study is consistent with the previous studies, that the efficacy of OCS is better as compared to RMI for differentiating adnexal masses. In our study, absent OCS was more sensitive (100 vs $55.6 \%$ ) but less specific than RMI ( 80.4 vs $95 \%$ ) as a predictor of malignancy. This is similar to the study by Yazbek et al which showed that negative OCS had better sensitivity 
than RMI (100 vs $89 \%$ ) but similar specificity of 93 vs $92 \% .{ }^{9}$ Kushtag et al also found OCS to be more sensitive (90.9 vs $72.7-82.8 \%$ ) but less specific (77.6\% vs 83.7 $89.9 \%$ ) than RMI. ${ }^{10}$ Combining OCS and RMI as well as sequential application was not found to be beneficial.

The weakness of the study may be that no borderline ovarian tumors were found, and so efficacy of OCS for diagnosis of borderline ovarian tumors cannot be commented upon.

\section{CONCLUSION}

Presence of ovarian crescent on ultrasound is highly indicative of benign nature of adnexal mass. Patients with absent OCS specially if $<10 \mathrm{~cm}$ in size, are likely to be malignant and should be triaged to higher centres for further treatment. OCS has the advantage of being less cumbersome, less dependent on operator's experience, quick, more accurate, inexpensive, non-calculative test with a good sensitivity and dependable specificity.

Funding: No funding sources Conflict of interest: None declared

Ethical approval: The study was approved by the Institutional Ethics Committee by Human Research (IECHR) of institution

\section{REFERENCES}

1. Morgante G, la Marca A, Ditto A, De Leo V. Comparison of two malignancy risk indices based on serum CA125, ultrasound score and menopausal status in the diagnosis of ovarian masses. J Obstet Gynaecol 1999;106:524-7.

2. Van Nagell JR Jr, Gershenson DM. Ovarian cancer: Etiology, Screening, and Surgery. In: Te Linde's Operative Gynaecology, $9^{\text {th }}$ ed. Rock JA, Jones HW, eds. Lippincott Williams \& Wilkins: Philadelphia; 2003:1487-522.

3. Manjunath AP, Pratapkumar, Sujatha K, Vani R. Comparison of three risk of malignancy indices in evaluation of pelvic masses. Gynecol Oncol 2001;81:225-9.
4. Suh-Burgmann E. Long-term outcomes following conservative surgery for borderline tumor of the ovary: a large population-based study. Gynecol Oncol. 2006;103:841-7.

5. Weiner Z, Thaler I, Beck D, Rottem S, Deutsch M, Brandes JM. Differentiating malignant from benign ovarian tumors with transvaginal color flow imaging. Obstet Gynecol. 1992;79:159-62.

6. Jacobs I, Oram D, Fairbanks J, Turner J, Frost C, Grudzinskas JG. A risk of malignancy index incorporating CA125, ultrasound and menopausal status for the accurate preoperative diagnosis of ovarian cancer. Br J Obstet Gynecol. 1990;97:922-9.

7. Hillaby K, Aslam N, Salim R, Lawrence A, Raju KS, Jurkovic D. The value of detection of normal ovarian tissue (the 'ovarian crescent sign') in the differential diagnosis of adnexal masses. Ultrasound Obstet Gynecol. 2004;23:63-7.

8. Tingulstad S, Hagon B, Skjeldestad FE, Onsrud M, Kiserud T, Halvorsen T, Nustad K. Evaluation of a risk of malignancy index based on serum CA125, ultrasound findings and menopausal status in the preoperative diagnosis of pelvic masses. $\mathrm{Br} \mathrm{J}$ Obstet Gynecol. 1996;103:826-31.

9. Yazbek J, Aslam N, Tailor A, Hillaby K, Raju KS, Jurkovic D. A comparative study of the risk of malignancy index and the ovarian crescent sign for the diagnosis of invasive ovarian cancer. Ultrasound Obstet Gynecol. 2006;28:320-4.

10. Kushtagi P, Kulkarni KK. Significance of the 'ovarian crescent sign' in the evaluation of adnexal masses. Singapore Med J. 2008;49(12):1017-20.

11. Van Holsbeke C, Van Belle V, Leone FP, Guerriero S, Paladini D, Melis GB, Greggi S, Fischerova D, De Jonge E, Neven P, Bourne T, Valentin L, Van Huffel $\mathrm{S}$, Timmerman D. Prospective external validation of the 'ovarian crescent sign' as a single ultrasound parameter to distinguish between benign and malignant adnexal pathology. Ultrasound Obstet Gynecol. 2010;36(1):81-7.

Cite this article as: Sharma A, Sharma R, Gulati A. Comparison of ovarian crescent sign and risk of malignancy index for prediction of ovarian malignancy in adnexal masses. Int J Reprod Contracept Obstet Gynecol 2021;10:541-5. 